Trends in der ästhetischen Dermatologie

\title{
Annual User Meeting Symposia L
}

Seit Eröffnung der deutschen LutronicNiederlassung im April 2017, ist es das primäre Ziel des Unternehmens, bestehenden und künftigen Kunden hochwertigen und schnellen Service zu bieten. Dies konnte im ersten Geschäftsjahr bereits erfolgreich umgesetzt werden: Die Fachärzte schätzen den individuellen Support, etwa im Bereich Training, Anwendung und Vermarktung.

Neben der Teilnahme an nationalen und internationalen Messen und Kon- gressen stehen sechs umfangreiche Workshops für 2018 auf dem Plan. Besonderes Highlight: das Annual User Meeting - Symposia L. Das Anwendertreffen richtet sich an Lutronic-Kunden aus ganz Europa. Nach dem erfolgreichen EuropaDebüt in der Schweiz 2017, startet Symposia L am 1. Juni 2018 auch in Deutschland (Kassel) durch. Die Veranstaltung bietet die Möglichkeit, von erfahrenen Anwendern zu lernen und sich mit anderen Fachärzten aktiv auszutauschen. Im
Zentrum des Programms stehen auch neue Forschungsergebnisse und Trends der ästhetischen Dermatologie. Um einen größtmöglichen Mehrwert für alle Teilnehmer zu erzielen, besteht die Möglichkeit, große Themenschwerpunkte vorzuschlagen, wie beispielsweise Handon-Trainings oder Fallbeispiele. Dies ist einzigartig auf dem deutschen Markt.

Interessierte können sich ab sofort bei Ralf Schmidt, National Sales Director Lutronic, telefonisch oder per Mail unter +49(0) 15121444502 oder ralf.schmidt@ lutronic.com für Symposia L, am 1. Juni 2018 in Kassel, vormerken.

Nach Informationen von Lutronic

\section{Microneedling zur Verbesserung des Hautbildes}

Erst knapp 18 Jahre sind seit der Erfindung des Dermarollers vergangen. Heutzutage wird der Dermaroller in unterschiedlichen Modellvarianten in eigener Manufaktur in Norddeutschland gefertigt und produziert und erfüllt als eines der wenigen Geräte seiner Klassifikation die strengen Auflagen medizinischer Zertifizierungen. Im Jahre 2013 wurde das Unternehmen Teil der mi.to. pharm $\mathrm{GmbH}$, deren Geschäftsführer Michael Tomerius über langjährige internationale Erfahrung in der Medizintechnik verfügt und dem Unternehmen neue Impulse gibt.

Die Dermaroller $\mathrm{GmbH}$, zertifiziert nach ISO 13485 und nach Anhang V der Medizinprodukterichtlinie 93/42/EEG, ist ein auf Mikronadeln spezialisierter Hersteller von Medizinprodukten. Die Dermaroller-Therapie, international bekannt

\section{Verbesserte Wundheilung nach Lasertherapie}

Einer Umfrage zufolge sind in Deutschland $15 \%$ der Erwachsenen tätowiert. Im Jahr 2016 nahmen nach Angaben der Deutschen Dermatologischen Lasergesellschaft rund 1,2 Millionen Personen die Möglichkeit wahr, die subkutanen Farbpigmente per Laserbehandlung entfernen zu lassen. Dieser Prozess verursacht auch oberflächliche Hautverletzungen. Wie eine in-vitro Studie zeigt, sind für die Behandlung laserinduzierter Wunden dexpanthenolhaltige Salben, wie Bepanthen ${ }^{\oplus}$ Wund- und Heilsalbe, der häufig angewendeten und empfohlenen Vaseline überlegen [Marquardt $\mathrm{Y}$ et al. Lasers in Surgery and Medicine 2015;47:257-65].

Um die Wundheilung in einem physiologischen Modell zu untersuchen, etablierte die Arbeitsgruppe von Prof. Jens Malte Baron ein dreidimensionales Wundheilungsmodell, bei dem ein $\mathrm{CO}_{2}$ Laser reproduzierbare, definierte Verletzungen von Epidermis und oberer Dermis verursacht. Für diese laserinduzierten Hautläsionen konnte bei topischer als Microneedling/Medical Needling, ist ein minimalinvasives Behandlungskonzept zur Verbesserung des Hautbildes ganz besonders bei Narben aller Art. Weitere Behandlungsfelder sind: Falten, Pigmentstörungen, Sonnenschäden der Haut, Schwangerschaftsstreifen etc. Die Dermaroller sind auch geeignet für das transdermale Einschleusen von Wirkstoffen.

In unserem Sortiment unterscheiden wir zwischen drei Modelllinien: Produkte für die rein medizinische Anwendung, Produkte für die Behandlung im Kosmetikinstitut und Produkte für den Heimgebrauch.

Nach Informationen von mi.to.pharm

Applikation von Bepanthen Wund- und Heilsalbe mit 5\% Dexpanthenol im 3DHautmodell eine bessere Wundheilung gezeigt werden als in Kontrollen, die mit Vaseline behandelt wurden.

Die Forschungsergebnisse im 3DHautäquivalent implizieren Konsequenzen für die ärztliche Praxis, denn Bepanthen Wund- und Heilsalbe könnte dem Patienten eine raschere Wundheilung nach der Laserbehandlung, etwa nach einer Tattoo-Entfernung, ermöglichen. „Wir haben gute Erfahrungen mit dexpanthenolhaltigen Salben in der Nachbehandlung der Lasertherapie gemacht und können deren Einsatz statt der üblichen Vaseline nur empfehlen“, resümiert Prof. Baron.

Nach Informationen von Bayer Vital 